\title{
Protein sequences classification by means of feature extraction with substitution matrices
}

\author{
Rabie Saidi 1,2,3,4, Mondher Maddouri4,5 and Engelbert Mephu Nguifo*1,2
}

\begin{abstract}
Background: This paper deals with the preprocessing of protein sequences for supervised classification. Motif extraction is one way to address that task. It has been largely used to encode biological sequences into feature vectors to enable using well-known machine-learning classifiers which require this format. However, designing a suitable feature space, for a set of proteins, is not a trivial task. For this purpose, we propose a novel encoding method that uses amino-acid substitution matrices to define similarity between motifs during the extraction step.

Results: In order to demonstrate the efficiency of such approach, we compare several encoding methods using some machine learning classifiers. The experimental results showed that our encoding method outperforms other ones in terms of classification accuracy and number of generated attributes. We also compared the classifiers in term of accuracy. Results indicated that SVM generally outperforms the other classifiers with any encoding method. We showed that SVM, coupled with our encoding method, can be an efficient protein classification system. In addition, we studied the effect of the substitution matrices variation on the quality of our method and hence on the classification quality. We noticed that our method enables good classification accuracies with all the substitution matrices and that the variances of the obtained accuracies using various substitution matrices are slight. However, the number of generated features varies from a substitution matrix to another. Furthermore, the use of already published datasets allowed us to carry out a comparison with several related works.
\end{abstract}

Conclusions: The outcomes of our comparative experiments confirm the efficiency of our encoding method to represent protein sequences in classification tasks.

\section{Background}

Analysis and interpretation of biological sequence data is a fundamental task in bioinformatics. Classification and prediction techniques are one way to deal with such task [1]. In fact, biologists are often interested in identifying the family to which a lately sequenced protein belongs [2]. This makes it possible to study the evolution of this protein and to discover its biological functions. Furthermore, the study and the prediction of oligomeric proteins (quaternary structures) are very useful in biology and medicine for many reasons [3]. Indeed, they often intervene in terms of bio-macromolecules functional evolution, reparation of misfolds and defects $[4,5]$. They are

\footnotetext{
* Correspondence: mephu@isima.fr

1 LIMOS - Blaise Pascal University - Clermont University, BP 10448, ClermontFerrand 63000, France

Full list of author information is available at the end of the article
}

also involved in many important biological processes such as chromosome replication, signal transduction, folding pathway and metabolism [6]. Biologists also seek, for instance, to identify active sites in proteins and enzymes [7], to classify parts of DNA sequences into coding or non-coding zones or to determine the function of the nucleic sequences such as the identification of the promoter sites and the junction sites [8-10].

Alignment is the main technique used by biologists to look for homology among sequences, and hence to classify new sequences into already known families/classes. Since relevant information is represented by strings of characters, this technique generally doesn't enable the use of well-known classification techniques such as decision trees (DT), naïve bayes (NB), support vector machines (SVM) and nearest neighbour (NN) which have proved to be very efficient in real data mining tasks [11]. In fact, 
those classifiers rely on data described in a relational format.

Meanwhile, different studies have been devoted to motif extraction in biological sequences [12-17]. Motifs extraction methods are generally based on the assumption that the significant regions are better preserved during the evolution because of their importance in terms of structure and/or function of the molecule [13], and thus that they appear more frequently than it is expected.

In [14], authors have shown that motif extraction methods can efficiently contribute to the use of machine learning algorithms for the classification of biological sequences. In this case, the classification obeys the knowl- edge discovery in data (KDD) process and hence comprises three major steps:

1. Preprocessing consists of extracting motifs from a set of sequences. These motifs will be used as attributes/features to construct a binary table where each row corresponds to sequence. The presence or the absence of an attribute in a sequence is respectively denoted by 1 or 0 . This binary table is called a learning context. It represents the result of the preprocessing step and the new sequence encoding format (figure 1).

2. In the mining step, a classifier is applied to the learning context to generate a classification model.

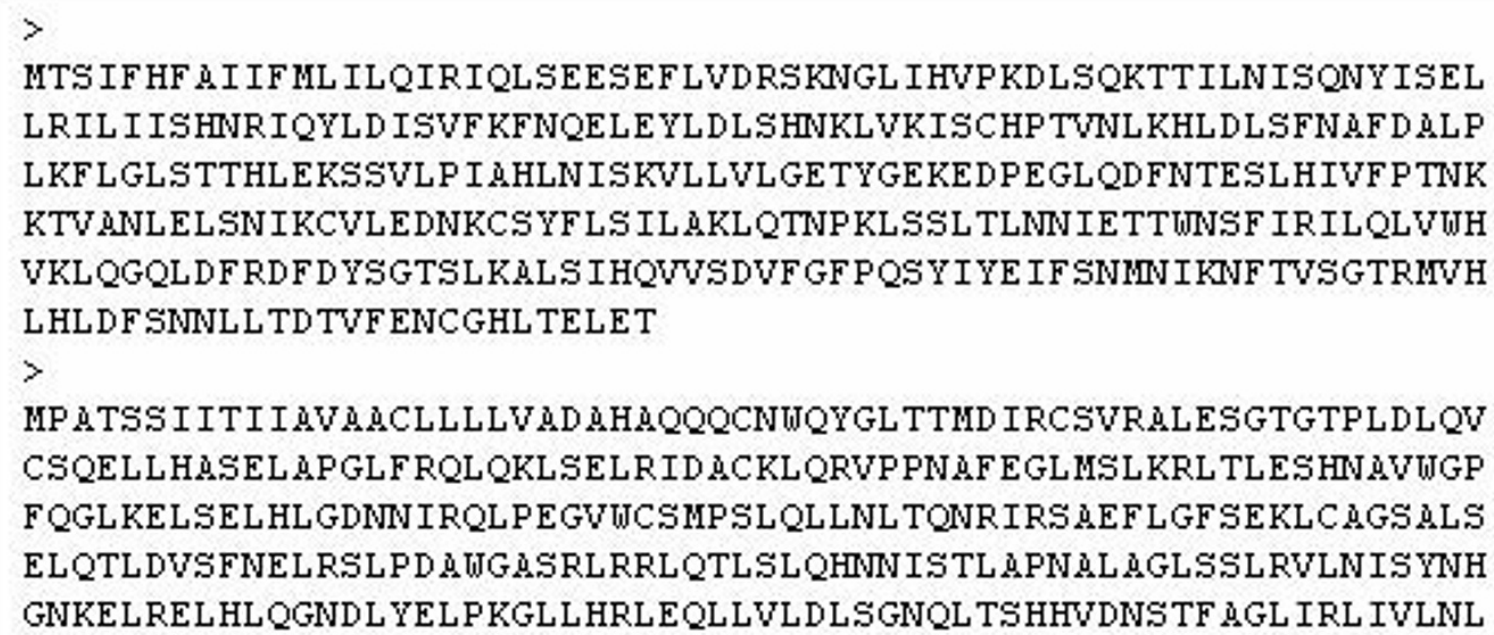

MTS IF HF A I IF ML ILQIR IQLSEESE F LVDRSKNGL I HVP KDLSQKTT ILN ISQNY ISEL LRIL I ISHNR IQYLD ISVF KFNQELE YLDLSHNKLVKISCHP TVNL KHLDLSFNAFDALP LKF LGLSTTHLEKSSVLP I AHLN ISKVLLVLGETYGE KEDPEGLQDFNTESLH IVF PTNK KTVANLELSNIKCVLEDNKCSYF LS IL AKLQTNP KLSSL TLNN IE TTWNSF IR ILQLVWH VKLQGQLDF RDF DYSGTSLKALS I HQVVSDVF GF PQSY I YE IF SNMN IKNF TVSGTRMVH LHLDFSNNLLTDTVF ENCGHLTELET $>$

MP AT TSS I ITI I AV Ä ACLLLLVAD ÄHAQQQCNWQYGLTTMD IRCSVRALLESGTGTPLDLQV

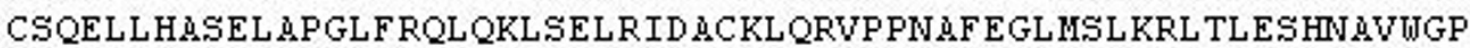
FQGL KELSELHLGDNN IRQL PE GVUCSMPSLQLLNL TQNR IRS AEF LGF SEKLCAGSALS ELQTLDVSFNELRSLPDAWGASRLRRLQTLSLQHNN ISTL APNAL AGLSSLRVLN ISYNH GNKELRELHLQGNDL YELPKGLL HRLEQLLVLDLSGNQL TSHHVDNSTF AGL IRL IVLNL

\section{$\int$ Attributes extraction}

\begin{tabular}{|l|}
\hline MPT \\
SRL \\
GVWT \\
AEF \\
$\ldots$ \\
\hline
\end{tabular}

Context construction

\begin{tabular}{|c|c|c|c|c|c|c|c|c|c|c|c|}
\hline & MPT & SRL & GVWT & AEF & TGF & HHV & KNF & LAK & AGL & SYN & DAL \\
\hline Seq1 & 1 & 0 & 0 & 0 & 1 & 0 & 1 & 1 & 0 & 1 & 0 \\
\hline Seq2 & 0 & 0 & 0 & 0 & 1 & 1 & 1 & 0 & 1 & 0 & 0 \\
\hline Seq3 & 1 & 0 & 1 & 0 & 0 & 1 & 0 & 0 & 0 & 1 & 1 \\
\hline Seq4 & 0 & 1 & 1 & 0 & 0 & 1 & 1 & 0 & 0 & 1 & 1 \\
\hline
\end{tabular}

Figure 1 Sequence pre-processing based on motif extraction. This figure describes the process of sequence encoding. The extracted motifs are used as attributes to build a binary context where each row represents a sequence. 
Table 1: Motifs clustering

\begin{tabular}{|c|c|c|c|c|c|c|c|c|c|}
\hline or & LLK & IMK & VMK & GGP & RI & RV & RF & RA & PP \\
\hline$P_{m}$ & 0.89 & 0.87 & 0.86 & 0 & 0.75 & 0.72 & 0.72 & 0.5 & 0 \\
\hline $\begin{array}{l}\text { Main } \\
\text { motif }\end{array}$ & LLK & LLK & LLK & GGP & $\mathrm{RI}$ & $\mathrm{RI}$ & $\mathrm{Rl}$ & $\mathrm{RV}$ & PP \\
\hline
\end{tabular}

$\mathrm{Cr}$ is a set of motifs (table 1) sorted by their lengths and $P_{m}$. The third row shows the cluster main motifs.

3. The latter model is used to classify other sequences in the post-processing step. These sequences are also encoded into a relational format using the same features as for the learning context i.e., test context.

In a previous work [18], we proposed a new method to encode protein sequences. It extends an existing method, termed Discriminative Descriptors (DD) [14], by taking into account the fact that some amino acids have similar properties and thus can be substituted by each other while changing neither the structure nor the function of the protein [19]. Hence, there might be several motifs that could be replaced by a single motif. We used amino acids substitution matrices to define such similarity; our encoding method is termed Discriminative Descriptors with Substitution Matrix (DDSM). Preliminary experiments conducted with $\mathrm{C} 4.5$ decision tree have shown promising results [18]. This manuscript presents a detailed experimental comparison (in terms of classification accuracy and number of attributes) between several encoding methods using various kinds of classifiers (C4.5 decision tree, NB, SVM and NN) as well as the standard approach based on alignment using Blast [20].

\section{Methods and Results}

\section{Some existing feature construction methods}

The following is a presentation of five existing methods of features construction: the N-Grams (NG), the Active Motifs (AM), the Amino Acid Composition (AAC), the Functional Domain Composition (FDC) and the Discrim-

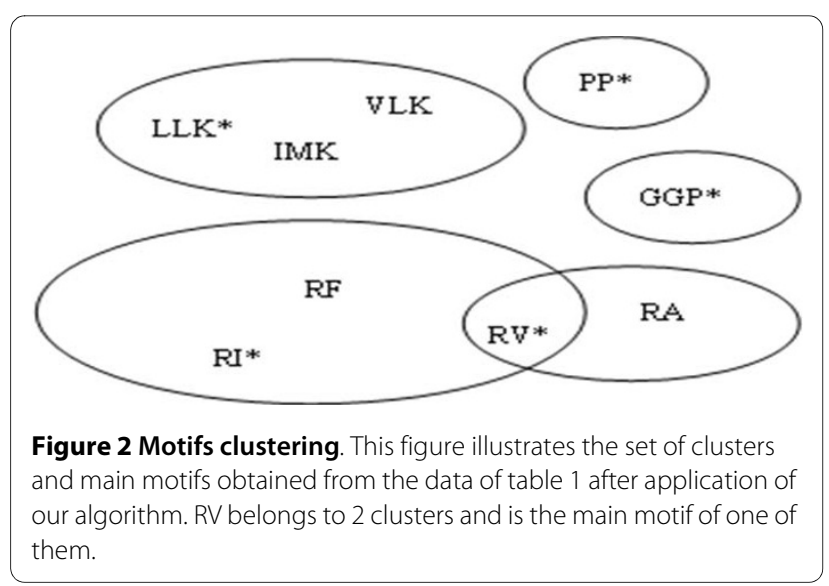

inative Descriptors (DD). After this, we re-describe our approach which consists of modifying the DD method by the use of a substitution matrix (DDSM) [18].

\section{$\mathrm{N}$-Grams}

The simplest approach is that of the N-Grams, known also as $\mathrm{N}$-Words or length $\mathrm{N}$ fenestration [21]. The motifs to be built have a predefined length. The $\mathrm{N}$-gram is a subsequence composed of $\mathrm{N}$ characters, extracted from a larger sequence. For a given sequence, the set of the Ngrams which can be generated is obtained by sliding a window of $\mathrm{N}$ characters on the whole sequence. This movement is carried out character by character. With each movement a subsequence of $\mathrm{N}$ characters is extracted. This process is repeated for all the analyzed sequences. Then, only the distinct $\mathrm{N}$-grams are kept.

\section{Active Motifs}

This method allows extracting the commonly occurring motifs whose lengths are longer than a specified length, called Active Motifs, in a set of biological sequences. The activity of a motif is the number of matching sequences given an allowed number of mutations [22]. The motif extraction is based on the construction of a Generalized Suffix Tree (GST) which is an extension of the suffix tree [23] and is dedicated to represent a set of $n$ sequences indexed each one by $\mathrm{i}=1 . . \mathrm{n}$.

\section{Amino Acid Composition}

According to the classic definition of this method, the feature set consists of 20 components, representing the 20 native amino acids in proteins. The amino acid composition refers to the occurrence frequency of each of these 20 components in a given protein. Since the information in the primary sequence is greatly reduced by considering the amino acid composition alone, other considerations have been taken into account within several studies such as the sequence-order correlation factors i.e., new features were added to the 20 original which yielded several AAC variants [24-28].

\section{Functional Domain Composition}

Biological databases, such as PFAM [29] and ASTRAL, contain large collections of multiple sequence alignments and Hidden Markov Model (HMM) profiles covering many common protein domains and families [29]. Functional domains are determined using computational 
Table 2: Experimental data

\begin{tabular}{|c|c|c|c|c|}
\hline Dataset (source) & $\begin{array}{l}\text { Identity percentage } \\
\text { (\%) }\end{array}$ & Family/class & Size & Total \\
\hline \multirow[t]{3}{*}{ DS1 (Swiss-prot) } & 48 & $\begin{array}{l}\text { High-potential Iron- } \\
\text { Sulfur Protein }\end{array}$ & 19 & 60 \\
\hline & & $\begin{array}{l}\text { Hydrogenase Nickel } \\
\text { Incorporation Protein } \\
\text { HypA }\end{array}$ & 20 & \\
\hline & & $\begin{array}{l}\text { Hlycine } \\
\text { Dehydrogenase }\end{array}$ & 21 & \\
\hline \multirow[t]{2}{*}{ DS2 (Swiss-prot) } & 48 & Chemokine & 255 & 510 \\
\hline & & Melanocortin & 255 & \\
\hline \multirow[t]{7}{*}{ DS3 (Swiss-prot) } & 25 & Monomer & 208 & 717 \\
\hline & & Homodimer & 335 & \\
\hline & & Homotrimer & 40 & \\
\hline & & Homotetramer & 95 & \\
\hline & & Homopentamer & 11 & \\
\hline & & Homohexamer & 23 & \\
\hline & & Homooctamer & 5 & \\
\hline \multirow[t]{2}{*}{ DS4 (Swiss-prot) } & 28 & human TLR & 14 & 40 \\
\hline & & Non-human TLR & 26 & \\
\hline \multirow[t]{4}{*}{ DS5 (SCOP) } & 84 & All-a domain & 70 & 277 \\
\hline & & All- $\beta$ domain & 61 & \\
\hline & & $\alpha / \beta$ domain & 81 & \\
\hline & & $a+\beta$ domain & 65 & \\
\hline
\end{tabular}

means, especially HMM profiles, combined with biologist knowledge and other databases information. Since they allow variable length gaps between several components, where each component is a simple motif $[15,16]$, functional domains can be considered as structured motifs. But they are more reliable since they obey the expert assessment.

\section{Descriminative Descriptors}

Given a set of $\mathrm{n}$ sequences, assigned to $\mathrm{P}$ families/classes $\mathrm{F}_{1}, \mathrm{~F}_{2} . ., \mathrm{F}_{\mathrm{P}}$, this method consists of building substrings called Discriminative Descriptors DD which allow to discriminate a family $F_{i}$ from other families $F_{j}$, with $i=1$..P and $\mathrm{i} \neq \mathrm{j}[14]$.

This method is based on an adaptation of the Karp, Miller and Rosenberg (KMR) algorithm [30]. This algorithm identifies the repeats in character strings, trees or tables. The extracted repeats are then filtered in order to keep only the discriminative and minimal ones.

A substring $\mathrm{X}$ is considered to be discriminative between the family $F_{i}$ and the other families $F_{j}$, with $i=$ 1..P, $\mathrm{j}=1 . . \mathrm{P}$ and $\mathrm{i} \neq \mathrm{j}$ if:

1. $\frac{\text { number of sequences of } F_{i} \text { where } X \text { appears }}{\text { total number of sequences of } F_{i}} \geq \alpha$

2. $\frac{\text { number of sequences of } F_{j} \text { where } X \text { appears }}{\text { total number of sequences of } F_{j}} \leq \beta$

where $\alpha$ and $\beta$ are user-specified thresholds between 0 and 1 .

\section{Proposed method: Discriminative Descriptors with Substitution Matrix}

In the case of protein, the Discriminative Descriptors method neglects the fact that some amino acids have similar properties and that they can be therefore substituted 
Table 3: Machine learning classifiers coupled with encoding methods

\begin{tabular}{|c|c|c|c|c|c|c|}
\hline \multicolumn{7}{|c|}{ Encoding method } \\
\hline Data & Mtr & Clfr & NG & AM & DD & DDSM \\
\hline \multirow[t]{5}{*}{ DS1 } & CA & C4.5 & 96.7 & 95 & 95 & 96.7 \\
\hline & & SVM & 96.7 & 93.3 & 96.7 & 96.7 \\
\hline & & NB & 86.7 & 90 & 81.7 & 80 \\
\hline & & NN & 63.3 & 78.3 & 60 & 61.7 \\
\hline & NA & & 4935 & 2060 & 4905 & 2565 \\
\hline \multirow[t]{5}{*}{ DS2 } & CA & C4.5 & 99.6 & 99.4 & 99.8 & 99.4 \\
\hline & & SVM & 100 & 99.4 & 100 & 100 \\
\hline & & NB & 100 & 74.7 & 100 & 100 \\
\hline & & NN & 100 & 100 & 100 & 98.8 \\
\hline & NA & & 6503 & 7055 & 10058 & 1312 \\
\hline \multirow[t]{5}{*}{ DS3 } & CA & C4.5 & $\underline{36.4}$ & - & $\underline{36.7}$ & 79.2 \\
\hline & & SVM & 43.2 & - & $\underline{43.2}$ & 78.94 \\
\hline & & NB & $\underline{43.2}$ & - & $\underline{43.1}$ & 59.4 \\
\hline & & $\mathrm{NN}$ & $\underline{20.9}$ & - & $\underline{21.3}$ & 77 \\
\hline & NA & & 7983 & - & 8403 & 508 \\
\hline \multirow[t]{5}{*}{ DS4 } & $C A$ & C4.5 & $\underline{60}$ & 57.5 & 77.5 & 82.5 \\
\hline & & SVM & 67.5 & 65 & 87.5 & 87.5 \\
\hline & & NB & 57.5 & 40 & 92.6 & 95 \\
\hline & & NN & 52.5 & 60 & 80 & 80 \\
\hline & NA & & 5561 & 3602 & 7116 & 5505 \\
\hline \multirow[t]{5}{*}{ DS5 } & $C A$ & C4.5 & 75.5 & 75.1 & 67.9 & 73.3 \\
\hline & & SVM & 84.1 & 81.2 & 82.3 & 82.3 \\
\hline & & NB & 77.3 & 63.7 & 84.5 & 85.9 \\
\hline & & NN & 80.5 & 79.4 & 78 & 78 \\
\hline & NA & & 6465 & 2393 & 13830 & 13083 \\
\hline
\end{tabular}

Mtr: Metric, Clfr: Classifier, CA: Classification Accuracy (\%), NA: Number of Attributes. 
by each other while changing neither the structure nor the function of the protein [19]. Indeed, we can find several motifs in the set of the attributes generated by the DD method, which are similar and can derive all from a single motif. In the same way, during the construction of the context (binary table), we are likely to lose information when we denote by 0 the absence of a motif while another one, that can replace it, already exists [18].

As mentioned, the similarity between motifs is based on the similarity between the amino acids which constitute them. Indeed, there are various degrees of similarity between amino acids. Since there are 20 amino acids, the mutations between them are scored by a $20 \times 20$ matrix called a substitution matrix $[19,21,31]$.

\section{Terminology}

Let $\mathrm{dr}$ be a set of $\mathrm{n}$ motifs, denoted each one by $\mathrm{dr}$ $[\mathrm{p}], \mathrm{p}=1 . . \mathrm{n}$. or can be divided into $m$ clusters. Each cluster contains a main motif $M^{*}$ and probably other motifs which can be substituted by $M^{*}$. The main motif is the one which has the highest probability of mutating to another in its cluster. For a motif $M$ of $k$ amino acids, this probability, noted $P_{m}(M)$, is based on the probability $P_{i}(i$
$=1 . . \mathrm{k})$ that each amino acid $M[i]$ of the motif $M$ does not mutate to any other amino acid. We have:

$$
P_{m}=1-\prod_{i=1}^{k} P_{i}
$$

$P_{i}$ is calculated based on the substitution matrix according to the following formula:

$$
P_{i}=S(M[i], M[i]) / \sum_{j=1}^{20} S^{+}\left(M[i], A A_{j}\right)
$$

$S(x, y)$ is the substitution score of the amino acid y by the amino acid $\mathrm{x}$ as it appears in the substitution matrix. $S^{+}(x, y)$ indicates a positive substitution score. $A A_{j}$ is the amino acid of index $j$ among the 20 amino acids.

For our purposes, a motif $M$ substitutes a motif $M^{\prime}$ if:

1. $M$ and $M^{\prime}$ have the same length $k$,

2. $S\left(M[i], M^{\prime}[i]\right)>=0, i=1 . . k$,

3. $S P\left(M, M^{\prime}\right)>=T$, where $T$ is a user-specified threshold such that $0<=T<=1$.

We denote by $S P(M, M)$ the substitution probability of the motif $M^{\prime}$ by the motif $M$ having the same length $k$. It measures the possibility that $M$ mutates to $M^{\prime}$ :

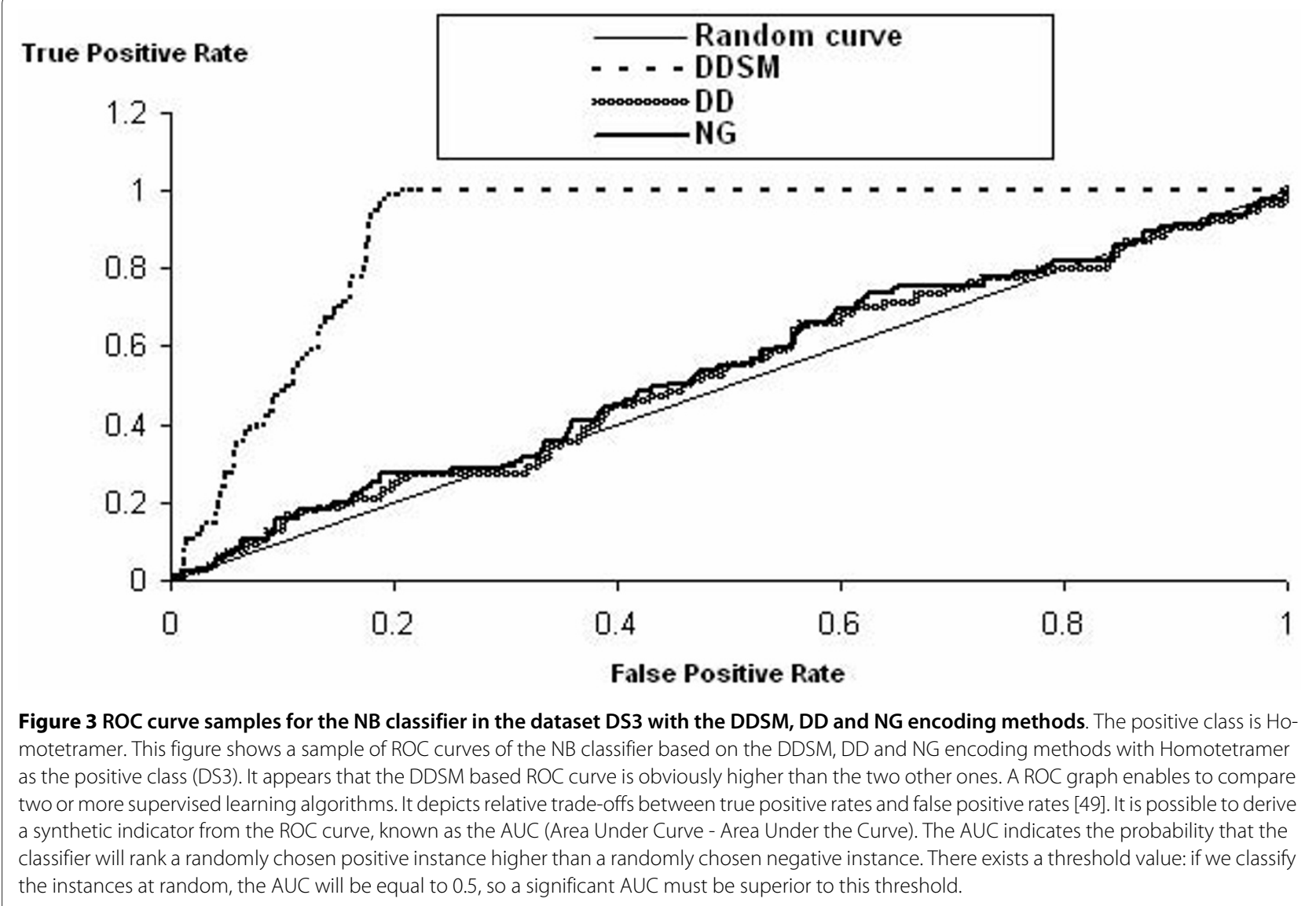


Table 5: Experimental results per substitution matrix for DS3

\begin{tabular}{llllll}
\hline \begin{tabular}{l} 
Substitution $\begin{array}{l}\text { Mttributes } \\
\text { matrix }\end{array}$ \\
\cline { 3 - 6 }
\end{tabular} & & & & Accuracy (\%) \\
\cline { 3 - 6 } & & C4.5 & SVM & NB & NN \\
\hline Blosum45 & 377 & 78.5 & 79.2 & 59.4 & 77.7 \\
Blosum62 & 508 & 79.2 & 78.9 & 59.4 & 77 \\
Blosum80 & 532 & 77.6 & 80.5 & 60 & 77.6 \\
Pam30 & 2873 & 77.8 & 82 & 60.3 & 76.7 \\
Pam70 & 802 & 78.1 & 80.5 & 60.5 & 77 \\
Pam250 & 1123 & 77.3 & 79.4 & 59.6 & 78.7 \\
\hline
\end{tabular}

$$
S P\left(M, M^{\prime}\right)=S_{m}\left(M, M^{\prime}\right) / S_{m}(M, M)
$$

$S_{m}(X, Y)$ is the substitution score of the motif $Y$ by the motif $X$. It is computed according to the following formula:

$$
S_{m}(X, Y)=\sum_{i=1}^{k} S(X[i], Y[i])
$$

It is clear, according to any substitution matrix, that there is only one best motif which can substitute a motif $M$. It is obviously itself, since the amino acids which constitute it are better substituted by themselves. This proves that the substitution probability of a motif by another one, if they satisfy the substitution conditions, will be between 0 and 1 .

\section{Methodology}

The encoding method is composed to two parts. First, the number of extracted motifs will obviously be reduced because we will keep only one motif for each cluster of substitutable motifs of the same length. Second, we will modify the context construction rule. Indeed, we will denote by 1 the presence of a motif or of one of its substitutes. The first part can be also divided into two phases: (1) identifying clusters' main motifs and (2) filtering. (1) The main motif of a cluster is the one that is the most likely to mutate to another in its cluster. To identify all the main motifs, we sort $\mathrm{Ch}$ in a descending order by motif lengths, and then by $P_{m}$. For each motif $M^{\prime}$ of $\mathrm{dr}$, we look for the motif $M$ which can substitute $M^{\prime}$ and that has the highest $P_{m}$ (probability of mutation to another motif). The clustering is based on the computing of the substitution probability between motifs. We can find a motif which belongs to more than one cluster. In this case, it must be the main motif of one of them. (2) The filtering consists of keeping only the main motifs and removing all

\begin{tabular}{|c|c|c|c|c|c|}
\hline $\begin{array}{l}\text { Substitution } \\
\text { matrix }\end{array}$ & Attributes & \multicolumn{4}{|c|}{ Accuracy (\%) } \\
\hline Blosum45 & 5095 & 82.5 & 85 & 95 & 80 \\
\hline Blosum80 & 5968 & 72.5 & 87.5 & 92.5 & 80 \\
\hline Pam30 & 7005 & 82.5 & 92.5 & 92.5 & 65 \\
\hline Pam70 & 5846 & 82.5 & 85 & 92.5 & 80 \\
\hline Pam 250 & 1948 & 82.5 & 77.5 & 95 & 80 \\
\hline
\end{tabular}

Table 6: Experimental results per substitution matrix for DS4 
Table 7: Experimental results per substitution matrix for DS5

\begin{tabular}{|c|c|c|c|c|c|}
\hline \multirow{2}{*}{$\begin{array}{l}\text { Substitution } \\
\text { matrix }\end{array}$} & \multirow[t]{2}{*}{ Attributes } & \multicolumn{4}{|c|}{ Accuracy (\%) } \\
\hline & & C4.5 & SVM & NB & NN \\
\hline Blosum45 & 12603 & 69.3 & 82.3 & 85.9 & 78 \\
\hline Blosum62 & 13083 & 73.3 & 82.3 & 85.9 & 78 \\
\hline Blosum80 & 13146 & 70.1 & 82.3 & 84.1 & 78 \\
\hline Pam30 & 13830 & 69.3 & 82.3 & 84.5 & 78 \\
\hline Pam70 & 13822 & 70.4 & 82.3 & 84.5 & 78 \\
\hline Pam250 & 1969 & 66.1 & 85.2 & 79.4 & 78 \\
\hline
\end{tabular}

the other substitutable ones. The result is a smaller set of motifs which can represent the same information as the initial set.

\section{Example}

Given a Blosum62 substitution matrix and the following set of motifs (table 1 ) sorted by their lengths and $P_{m}$, we assign each motif to a cluster represented by its main motif. We get 5 clusters illustrated by the diagram shown in figure 2.

\section{Experimental environment}

NG, AM, DD and DDSM encoding methods are implemented in $C$ language and gathered into a DLL library (additional file 1). The accepted format of the input files is the FASTA format for biological sequences files. The library code that we have implemented generates relational files under various formats such as the ARFF format used by the workbench WEKA [32] and the DAT format used by the system DisClass [14].

Our experiments are divided into 2 parts. In the first one, we make a detailed comparison between NG, AM, DD and DDSM encoding methods to confirm the results obtained in [18]. We perform the sequence classification using DT, SVM, NB and NN algorithms as described in section 1. We also conducted classification experiments using Blast [20] i.e., we assign to a protein query the class with the best hit score. Our method (DDSM) constructs the features using the substitution matrix Blosum62. The choice of this substitution matrix is not based on preliminary experiments, but instead on the fact that it is the most used by alignment tools especially the widespread Blast. We examine three aspects:

1. The effect of each encoding method on the four classifiers to deduce which one is the best in terms of accuracy and number of generated attributes.

2 . The comparison of the four classifiers while varying the encoding methods.

3. The comparison with Blast results.

In the second part, we try to assess the effect of varying the substitution matrices on our method and on the classification quality and hence to determine whether there is a substitution matrix which could be recommended. Then we compare our feature-construction method with other ones presented in $[27,28,33]$, which means that we compare with nine related works [27,28,33-39].

\section{Part 1}

To perform our experiments, we use 5 datasets comprising 1604 protein sequences from Swiss-Prot [40] and SCOP [41] described in table 2 (additional file 2).

Table 8: Comparison with results reported in (Yu et al., 2006) for DS3

\begin{tabular}{|c|c|c|}
\hline Methods & Accuracy \% & Correctly classified sequences \\
\hline DDSM \& C4.5 & 79.2 & 568 \\
\hline DDSM \& SVM & 78.9 & 588 \\
\hline DDSM \& NB & 59.4 & 434 \\
\hline DDSM \& NN & 77 & 564 \\
\hline FDC \& NN & 75.2 & 539 \\
\hline$A A C \& N N$ & 41.4 & 297 \\
\hline Blast-based & 69.6 & 499 \\
\hline
\end{tabular}


Table 9: Comparison with results reported in (Chen et al., 2006) and (Zhou, 1998) for DS5

\begin{tabular}{lll}
\hline Methods & Accuracy \% & Correctly classified sequences \\
\hline DDSM \& C4.5 & 73.3 & 203 \\
DDSM \& SVM & 82.3 & 228 \\
DDSM \& NB & 85.9 & 238 \\
DDSM \& NN & 78 & 216 \\
Blast-based & 78.3 & 220 \\
AAC[27] \& SVM [27] & 80.5 & 223 \\
pair-coupled AAC[27] \& SVM [27] & 77.6 & 215 \\
PseAAC[27] \& SVM [27] & 80.5 & 223 \\
SVM fusion [27] & 87.7 & 243 \\
AAC[28] \& Component coupled [28] & 79.1 & 219 \\
AAC[34] \& City-block distance [34] & 59.9 & 166 \\
AAC[35] \& Euclidean distance [35] & 55.2 & 153 \\
AAC[36] \& Neural network [36] & 74.7 & 206 \\
AAC[37] \& SVM [37] & 79.4 & 219 \\
AAC[38] \& LogitBoost [38] & 84.1 & 233 \\
AAC[39] \& Rough Sets [39] & 79.4 & 219 \\
\hline
\end{tabular}

We try to conduct our experiments on various kinds of datasets. These datasets differ from one another in terms of size, number of class, class distribution, complexity and sequence identity percentage. The first dataset DS1 contains 3 distinct and distant protein families. We suppose that classification in this case will be relatively easy since each family will probably have preserved patterns which are different from those of other families [13]. DS2 represents a bigger dataset comprising two sub-families of protein sequences belonging to the Rhodopsin Like/ Peptide family. However, the datasets DS3 and DS4 present more difficult classification problems. DS3 contains seven classes that represent seven categories of quaternary (4D) protein structure with a sequence identity of $25 \%$. The problem here lies in recognizing the $4 \mathrm{D}$ structure category from the primary structure. In this case, an important question is to be answered: does the primary structure contain sufficient information to identify the 4D structure? The task relative to DS4 is that of distinguishing between the human Toll-like Receptors (TLR) protein sequences and the non-human ones. The difficulty is due to the structural and functional similarity of the two groups. The choice of this dataset came after Biologists of Pasteur Institute of Tunis (PIT) asked to help them in identifying TLR families especially human ones among the 40 TLR that exist. DS5 consists of 277 domains: 70 all- $\alpha$ domains, 61 all- $\beta$ domains, $81 \alpha / \beta$ domains, and $65 \alpha+\beta$ domains from SCOP [41]. This challenging dataset was constructed by Zhou [28] and has been extensively used to address structural class prediction [27,28,34-39].

\section{Part 2}

In this part, we consider again the datasets DS3, DS4 and DS5 since they are considered to be delicate classification tasks and can thus reveal valuable information about the efficiency of the classifiers and the feature-construction methods. We try to investigate the effect of the substitution matrices variation on the quality of our encoding method and hence on the classification quality using C4.5, SVM, NB and NN algorithms. We employ all the substitution matrices used by the standalone version of Blast and belonging to the two well-known families: Blosum [19] and Pam [42] i.e., Blosum45, Blosum62 Blosum80, Pam30, Pam70, Pam 250.

Since DS3 is the same dataset as in [33], these experiments allow us to compare our encoding method with other related ones presented in that paper, where the nearest neighbour algorithm NN was coupled with each of the following methods: functional domain composition FDC, amino acid composition AAC and Blast alignment tool [20], to predict the quaternary structures categories of the proteins. In fact, the investigation of the quaternary structures prediction using computational tools remains a task with important implications for many reasons. First, these structures are involved in many biological processes and have direct link with known diseases like sickle-cell anaemia. Second, the in vitro methods are very slow and costly in spite of being 
accurate. This comparison allows us to assess whether our feature-construction method could offer any benefits over the above-mentioned methods quoted in [33] while using the same classifier (NN) and learning technique (leave-one-out).

Since prior information on the structure of a protein can provide useful information about its function, many other works similar to [33] have investigated this topic [27,28,34-39,43-46]. These works often use kinds of amino acid composition or functional domain composition to deal with the prediction of oligomeric proteins or protein structural classes. DS5 represents a challenging dataset that has been extensively used to address structural class prediction [27]. This allows us to compare our method with several works existing in the literature.

\section{Discussion and Conclusions}

\section{Experimental Techniques}

The computations are carried out on a computer with an Intel Centrino 1.6 GHz CPU and 1Go of main memory. Results are shown in the next sub-sections tables. Best accuracies, for each dataset, are shown in bold and results below minimum accepted values results are underlined. The minimum accepted value (MAV) is obtained by assigning all the sequences of a dataset to its biggest class. Hence, we have $35 \%, 50 \%, 46.7 \%, 65 \%$ and $29.2 \%$ as MAVs respectively for DS1, DS2, DS3, DS4 and DS5. We also show the number of attributes generated by each method.

In the classification process, we use the leave-one-out technique [11] also known as jack-knife test. For each dataset (comprising $\mathrm{n}$ instances), only one instance is kept for the test and the remaining part is used for the training. This action is repeated $\mathrm{n}$ times. The leave-oneout is considered to be the most objective test technique compared to the other ones i.e., hold-out, $n$-cross-validation. Indeed the leave-one-out test allows to obtain the same classification results regardless of the number of runs, which is not the case for the other tests (see the monograph [47] for the mathematical principle and [48] for a comprehensive discussion). For the encoding methods, we use default parameters as in [18]: NG $(\mathrm{N}=3)$, $\mathrm{AM}$ (min-length $=3$, activity $=25 \%)$, DD and DDSM $(\alpha=$ $0, \beta=0$ except for DS3 where $\beta=1$ to reduce the runtime), DDSM (substitution matrix $=$ Blosum62, substitution probability threshold $\mathrm{T}=0.9$ ). These parameters can also be specified by users.

We recall that in part 1 , we use the following classifiers: C4.5 decision tree, support vector machine SVM, naïve bayes NB and nearest neighbour algorithm $\mathrm{NN}$ of the workbench WEKA [32]. We generate and test the classification models; then we report the classification accuracy (rate of correctly classified sequences). Moreover, we conducted the leave-one-out test on the same datasets using Blast as already explained in section 2.3. In part 2, we investigate any potential effect of the substitution matrix variance on the features building and the classification quality, and then we compare it with other classification systems quoted in $[27,28,33]$.

\section{Part 1 Results}

The experimental results vary according to the input data (table 3 and table 4). The classification of the datasets DS1 and DS2 was relatively easy, as expected. Each family probably has its own motifs which characterize it and distinguish it from the others. This explains the high accuracies reached by all the classifiers with all the encoding methods. But it is notable that the N-Grams encoding gave the best results although it is the simplest method to use. Moreover, since this kind of classification, is easy, it does not require any sophisticated preprocessing and can simply be addressed by using alignment tools; indeed Blast arrived at full accuracy (table 4).

As for DS3, classification represents a real challenge. In fact, it is comprised of 717 sequences unequally distributed into seven classes which represent seven quaternary protein structure categories. It is a question of predicting the $4 \mathrm{D}$ structure based only on the primary structure without any complementary information. The AM method could not be used because it generates a great number of attributes (dashes in table 3). The obtained accuracies with the NG and the DD methods were below the MAV (within 20.9\% and 43.2\%) and the result obtained by Blast was acceptable $(69.60 \%)$ while the best accuracy reached $(79.2 \%)$ was obtained with the DDSM method (figure 3 illustrates a sample of ROC curves [49] of the NB classifier based on the DDSM, DD and NG encoding methods with Homotetramer as the positive class from DS3).

The dataset DS4 was not as easy to classify as DS1 and DS2 since the human TLR and the non-human TLR resemble each other in terms of function and structure. Indeed the two classes share many similar parts, making it difficult to discriminate them. That is why alignment based classification (using Blast) didn't reach full accuracy as it did for the two first datasets. The NG and the AM encoding seems to be inefficient since they gave accuracies below the MAV with two classifiers. The DD method outperforms the two previous methods (NG and AM). Since it adopts a discriminating approach to build the attributes, it allowed a better distinction between the human TLR and the non-human TLR. But, to improve classification in the dataset DS4, it is necessary to take into account the phenomenon of mutation and substitution between the amino acids which constitute the protein sequences. Indeed, the DDSM method made it possible to reach the highest precisions with all the classifiers, while reducing the number of generated attributes. Experimental results obtained with DS5 show a good per- 
formance for all the encoding methods, though no full accuracy was reached. We can notice that NG performed very well and allowed to improve results with the classifiers C4.5, SVM and NN. Blast allowed also to obtain good accuracy which is due to the high identity percentage within the dataset. But, the best accuracy was obtained with DDSM $(\approx 86 \%)$.

Moreover, we can notice that SVM generally provided the best accuracies with all the encoding methods, though it is known as a slow classifier. So, we can conclude that the combination (DDSM, SVM) could be an efficient system for the protein sequences classification.

\section{Part 2 results}

In this section, we study the effect of the substitution matrices (SM) variation on the classification by applying some of the most often used SMs belonging to the two well-known families: Blosum and Pam [19,42]. These SMs are the same used by the standalone version of Blast [20].

Substitution scoring is based on the substitution frequencies seen in multiple sequence alignments. Yet it differs from Pam to Blosum. Whereas the Pam matrices have been developed from global alignments of closely related proteins, the Blosum matrices are based on local multiple alignments of more distantly related sequences. This would have an effect on the representation size. Indeed, the number of constructed features varies from a substitution matrix to another. Blosum matrices with low numbers and Pam matrices with higher numbers allow the building of fewer features since they score highly the substitution between amino acids. This would yield larger clusters of substitutable motifs, and hence fewer main motifs i.e., fewer features (see section 2.2.2 and 2.2.3).

However, the variances of accuracies are slight when varying the substitution matrices with the same classifier (table 5, table 6 and table 7). Moreover, no substitution matrix allows obtaining the best accuracy for all the classifiers. We can even notice contradicting results; indeed, in DS3 and DS4, NN algorithm performs worse when coupled with Pam30, while the same matrix allows SVM to reach its best accuracy. The same phenomenon is noticed in DS5 with the classifiers C4.5 and SVM and the matrix Pam250. If one looks for reduced-size representation, Blosum matrices with low numbers and Pam matrices with higher numbers are recommended.

Since we used the same dataset (DS3) and the same assessment technique (leave-one-out) as in [33], we compare our feature-building method (DDSM with default parameter values: $\alpha=0, \beta=0$, substitution matrix $=$ Blosum62, substitution probability threshold $\mathrm{T}=0.9$ ) with the ones studied in [33] (FDC, AAC, and Blast coupled each one with the nearest neighbor algorithm NN). Comparative results are reported in table 8 . We can notice that the worst results were obtained with the AAC method. Indeed, the obtained results were below the MAV $46.7 \%$. Blast arrived at better results, but the accuracy was not very high. In fact, an analysis of the Protein Data Bank (PDB) [50], where the protein structures are deposited, reveals that proteins with more than $30 \%$ pairwise sequence identity have similar 3D structures [51]. But in our case we process a dataset with a sequence identity of $25 \%$. The FDC method seems to be promising since it allowed reaching an accuracy of $75.2 \%$. But our method was quite better and enabled to reach the highest accuracy rates among the mentioned methods and also coupled with the same classifier i.e., NN algorithm (77\%).

If we look for better classification systems we can consider the combinations (DDSM \& C4.5) or (DDSM \& SVM). In addition higher accuracy can be obtained by using the combination (DDSM \& SVM) and the matrix Pam30 which enabled to reach an accuracy of $82 \%$ (table 8). This indicates that SVM coupled with our encoding method DDSM represent an efficient system for protein classification.

In the same way, the use of the same dataset (DS5) and the same validation technique (leave-one-out) as in $[27,28]$ allowed us to compare our method with these two works as well as six others [34-39]. In these studies, variants of the amino acid composition AAC have been proposed to encode protein sequences and then coupled with a classifier to predict the protein structural classes. These works are based in the assumption that there is a strong correlation between the AAC and the structural class of a protein. In table 9, we report the results obtained by our method (DDSM with default parameter values: $\alpha=0, \beta=0$, substitution matrix $=$ Blosum62, substitution probability threshold $\mathrm{T}=0.9$ ) coupled with C4.5, SVM, NB and NN as well as the results of the related works (in table 9, $\mathrm{AAC}_{\mathrm{x}}$ means the AAC variant presented in the paper $\mathrm{x}$ ). We can claim that our encoding method generally outperforms any AAC encoding method proposed by the above-mentioned works. In [27], authors coupled three kinds of AAC with SVM i.e., (AAC \& SVM), (pair-coupled AAC \& SVM) and (PseAAC \& SVM). In the best case, they reached an accuracy of $80.5 \%$, whereas the combinations (DDSM \& SVM) and (DDSM \& NB) allowed reaching respectively $82.3 \%$ and $85.9 \%$ of accuracy. To enhance their results, authors in [27] proposed a fusion network that combines the results obtained by the three proposed combinations and they arrived at an accuracy of $87.7 \%$. Although, this result is slightly superior to ours, it does not mean that their encoding method outperforms DDSM. Indeed, the improvement of their results comes from the fusion network classifier and not from the AAC variants they use. Moreover, in most of these related works [27,28,34-39], authors perform a fine-tuning to look for the classifier 
parameter values allowing to get the best results, whereas we just use the default parameter values of both our encoding method and the classifiers as found in WEKA [32]. This fine tuning allowed to reach competitive accuracies which is the case of the combination (AAC \& LogitBoost) [38]. We believe that we can also reach higher accuracies if we perform a fine-tuning of the parameters of our method and the classifiers. But, we chose to just use the default parameter values to make it easier for users who may have no prior knowledge on what these parameters mean or how to specify them.

\section{Additional material}

Additional file $\mathbf{1}$ Software, $\mathbf{1 8 5} \mathrm{K}$. SeqCod.zip contains programs which are also available upon request from the authors or from http:// www.cril.univ-artois.fr/ mephu/SegCod.

Additional file 2 Experimental data, $328 \mathrm{~K}$. Experimental_data.zip comprises all datasets with their classification files.

Competing interests

The authors declare that they have no competing interests.

\section{Authors' contributions}

RS participated in the design of the DDSM method, implemented the programs, selected data, carried out the experimental study, performed the statistical analysis and drafted and revised the manuscript. MM conceived the DD method, participated in DDSM design, participated in the data selection and revised the manuscript. EMN conceived the DDSM method, provided assistance in the statistical analysis and revised the manuscript. All authors read and approved the final manuscript.

\section{Acknowledgements}

This work was partially supported by the French-Tunisian project CMCU-Utique 05G1412 and LifeGrid Prefon_Meta project. We would like to thank JeanJacques Givry, Alia Bouali, Philippe Fournier-Viger, Shannon Behary and Lamia Nammouchi for English proofreading. We also would like to thank Sabeur Aridhi, Manel Zoghlami and Wajdi Dhifli for technical help.

\section{Author Details}

1 LIMOS - Blaise Pascal University - Clermont University, BP 10448, ClermontFerrand 63000, France, 2LIMOS - CNRS UMR 6158, Aubière 63173, France, 3Department of Computer Science - FSJ - University of Jendouba, UMA Street, Jendouba 8100, Tunisia, 4URPAH - FST - University of Tunis El Manar, Academic Campus, Tunis 2092, Tunisia and 5 Department of Computer Science - FSG University of Gafsa, Campus of Sidi Ahmed Zarroug, Gafsa 2112, Tunisia

Received: 4 September 2009 Accepted: 8 April 2010

Published: 8 April 2010

\section{References}

1. Bhaskar H, Hoyle DC, Singh S: Machine learning in bioinformatics: A brief survey and recommendations for practitioners. Computers in Biology and Medicine 2005, 36:1104-1125.

2. Bernardes J, Fernandez J, Vasconcelos A: Structural descriptor database: a new tool for sequence based functional site prediction. $B M C$ Bioinformatics 2008, 9:492.

3. Klotz C, Aumont MC, Leger JJ, Swynghedauw B: Human cardiac myosin ATPase and light subunits: A comparative study. Biochim Biophys 1975, 386:461-469

4. Price NC: Assembly of multi-subunit structure. In Mechanisms of Protein Folding Edited by: Pain RH. Oxford University Press, New York; 1994:160-193.

5. Corrales FJ, Fersht AR: Kinetic significance of $\mathrm{GroEL}_{14} \cdot\left(\mathrm{GroES}_{7}\right)_{2}$ complexes in molecular chaperone activity. Folding \& Design 1996, 1:265-273.
6. Terry BF, Richard MC: Determination of protein-protein interactions by matrix-assisted laser desorption/ionization mass spectrometry. J Mass Spectrom 1998, 33:697-704.

7. Slama P, Filippis I, Lappe M: Detection of protein catalytic residues at high precision using local network properties. BMC Bioinformatics 2008, 9:517.

8. Mephu Nguifo E, Sallantin J: Prediction of Primate Splice Junction Gene Sequences with a Cooperative Knowledge Acquisition System. ISMB 1993:292-300

9. Lemoine E, Merceron D, Sallantin J, Mephu Nguifo E: Improving the Efficiency of a User-Driven Learning System with Reconfigurable Hardware. Application to DNA Splicing. Pacific Symposium on Biocomputing 1999:290-301.

10. Vignal L, D'Aubenton-Carafa Y, Lisacek F, Mephu Nguifo E, Rouze P, Quinqueton J, Thermes C: Exon prediction in eucaryotic genomes. Biochimie 1997, 78:327-334.

11. Han J, Kamber M: Data Mining: Concepts and Techniques. Morgan Kaufmann Publishers; 2006.

12. Huan L, Motoda H: Feature Extraction, Construction and Selection: A Data Mining Perspective. ISBN: 978-0-7923-8196-9. Kluwer Academic Publishers, Norwell, MA; 1998.

13. Nevill-Manning CG, Wu TD, Brutlag DL: Highly specific protein sequence motifs for genome analysis. Proceedings of the National Academy of Sciences of the United States of America 1998, 95(11):5865-5871.

14. Maddouri M, Elloumi M: Encoding of primary structures of biological macromolecules within a data mining perspective. Journal of Computer Science and Technology (JCST) 2004, 19(1):78-88.

15. Yongqiang Z, Zaki MJ: EXMOTIF: efficient structured motif extraction. Journal of Algorithms for Molecular Biology 2006, 1:21.

16. Yongqiang Z, Zaki MJ: SMOTIF: efficient structured pattern and profile motif search. Journal of Algorithms for Molecular Biology 2006, 1:22.

17. Lopes F, Martins D, Cesar R: Feature selection environment for genomic applications. BMC Bioinformatics 2008, 9:451

18. Saidi R, Maddouri M, Mephu Nguifo E: Biological Sequences Encoding for Supervised Classification. Intl. conf. on Biolnformatics Research and Development (BIRD), TU Berlin, March 12-14, Springer-Verlag, LNBI 4414 2007:224-238

19. Henikoff S, Henikoff JG: Amino acid substitution matrices from protein blocks. National Academy of Sciences, USA 1992, 89:10915-10919.

20. Altschul SF, Gish W, Miller W, Myers EW, Lipman DJ: Basic local alignment search tool. Journal of Molecular Biology 1990, 215(3):403-413.

21. Leslie $C$, Eskin $E$, Noble WS: The spectrum kernel: a string kernel for svm protein classification. Pacific Symposium on Biocomputing (PSB;) 2002:564-575

22. Wang JTL, Marr TG, Shasha D, Shapiro BA, Chirn GW: Discovering active motifs in sets of related protein sequences and using them for classification. Nucleic Acids Research 1994, 22(14):2769-2775.

23. Hui LCK, Crochemore M, Galil Z, Manber U: Combinatorial Pattern Matching. Lecture Notes in Computer Science in Apostolico, Springer-Verlag 1992, 644:230-243.

24. Zhang CT, Chou KC, Maggiora GM: Predicting protein structural classes from amino acid composition: Application of fuzzy clustering. Protein Eng 1995, 8:425-435.

25. Chou KC, Cai YD: Predicting protein quaternary structure by pseudo amino acid composition. Proteins 2003, 53:282-289.

26. Zhang SW, Pan Q, Zhang HC, Zhang YL, Wang HY: Classification of protein quaternary structure with support vector machine. Bioinformatics 2003, 19:2390-2396.

27. Zhou GP: An intriguing controversy over protein structural class prediction. J Protein Chem 1998, 17:729-738.

28. Finn RD, Tate J, Mistry J, Coggill PC, Sammut JS, Hotz HR, Ceric G, Forslund K, Eddy SR, Sonnhammer EL, Bateman A: The Pfam protein families database. Nucleic Acids Research 2008, 36:D281-D288.

29. Karp R, Miller RE, Rosenberg AL: Rapid Identification of Repeated Patterns in Strings, Trees and Arrays. 4th Symposium of Theory of Computing 1972:125-136.

30. Malde $K$ : The effect of sequence quality on sequence alignment. Bioinformatics 2008, 24(7):897-900

31. Witten IH, Eibe F: Data Mining: Practical machine learning tools and techniques. 2nd edition. Morgan Kaufmann, San Francisco; 2005.

32. Yu X, Wang C, Li Y: Classification of protein quaternary structure by functional domain composition. BMC Bioinformatics 2006, 7:187-192. 
33. Chou PY: Prediction of protein structural classes from amino acid composition. In Prediction of Protein Structure and the Principles of Protein Conformation Edited by: Fasman GD. Plenum Press, New York; 1989:549-586.

34. Nakashima H, Nishikawa K, Ooi T: The folding type of a protein is relevant to the amino acid composition. J Biochem 1986, 99:152-162.

35. Cai YD, Liu XJ, Xu XB, Zhou GP: Support vector machines for predicting protein structural class. BMC Bioinform 2001, 2:1-5.

36. Cai YD, Zhou GP: Prediction of protein structural classes by neural network. Biochimie 2000, 82:783-785.

37. Feng KY, Cai YD, Chou KC: Boosting classifier for predicting protein domain structural class. Biochem Biophys Res Commun 2005, 334:213-217.

38. Cao YF, Liu S, Zhang LD, Qin J, Wang J, Tang KX: Prediction of protein structural class with Rough Sets. BMC Bioinform 2006, 7:

39. Bairoch A, Apweiler R: The SWISS-PROT protein sequence database and its supplement TrEMBL in 2000. Nucleic Acids Research 2000, 28(1):45-48.

40. Andreeva A, Howorth D, Brenner SE, Hubbard TJP, Chothia C, Murzin AG: SCOP database in 2004: refinements integrate structure and sequence family data. Nucl Acid Res 2004, 32:D226-D229.

41. Dayhoff MO, Schwartz RM, Orcutt BC: A Model of Evolutionary Change in Proteins. National Biomedical Research Foundation, Washington DC 1978, 5:345-352.

42. Zhang SW, Pan Q, Zhang HC, Shao ZC, Shi JY: Prediction protein homooligomer types by pesudo amino acid composition: approached with an improved feature extraction and Naive Bayes feature fusion. Amino Acids 2006, 30:461-468.

43. Song J, Tang H: Accurate classification of homodimeric vs other homooligomeric proteins using a new measure of information discrepancy. J Chem Inf Comput Sci 2004, 44:1324-1327.

44. Chou KC: Prediction of protein structural classes and subcellular locations. Curr Protein Peptide Sci 2000, 1:171-208.

45. Chou KC, Cai YD: Predicting protein structural class by functional domain composition. Biochem Biophys Res Commun 2004, 321:1007-1009

46. Mardia KV, Kent JT, Bibby JM: Multivariate analysis. London: Academic Press; 1979:322-381.

47. Chen C, Zhou XB, Tian YX, Zhou XY, Cai PX: Predicting protein structural class with pseudo-amino acid composition and support vector machine fusion network. Anal Biochem 2006, 357:116-121.

48. Chou KC, Zhang CT: Review: Prediction of protein structural classes. Crit Rev Biochem Mol Biol 1995, 30:275-349.

49. Zweig MH, Campbell G: Receiver-operating characteristic (ROC) plots: a fundamental evaluation tool in clinical medicine. Clinical chemistry 1993, 39(8):561-577.

50. Berman HM, Westbrook J, Feng Z, Gilliland G, Bhat TN, Weissig H, Shindyalov IN, Bourne PE: The Protein Data Bank. Nucleic Acids Res 2000, 28(1):235-242.

51. Sander C, Schneider R: Database of homology derived protein structure and the structural meaning of sequence alignment. Protein: Structure, Function and Genetic 1991, 9:56-68.

\section{Submit your next manuscript to BioMed Central} and take full advantage of:

- Convenient online submission

- Thorough peer review

- No space constraints or color figure charges

- Immediate publication on acceptance

- Inclusion in PubMed, CAS, Scopus and Google Scholar

- Research which is freely available for redistribution 